\title{
Base-promoted acetal formation employing aryl salicylates
}

\author{
Pinmanee Boontheung, Patrick Perlmutter*, and Evaloni Puniani \\ School of Chemistry, Monash University, PO Box 23, Victoria 3800 Australia \\ E-mail: Patrick.perlmutter@sci.monash
}

\section{Dedicated to Professor T. R. Govindachari on the occasion of his $85^{\text {th }}$ birthday \\ (received 21 May 01; accepted 16 Sep 01; published on the web 24 Sep 01)}

\begin{abstract}
New examples of base-promoted acetal formation in the reactions of aryl salicylate and salicylate-type esters 1 to 3 with aldehydes are reported.
\end{abstract}

Keywords: Oxo-acetals, aldehydes, salicylate, DABCO

\section{Introduction}

Recently we reported that aryl esters of 2-hydroxybenzoic acids (i.e. salicylates) form cyclic acetals with a variety of aldehydes (scheme 1) under base-catalyzed conditions. ${ }^{1}$ Key aspects of the process were that the reactions worked best under neat conditions or, where necessary, with minimal solvent to effect dissolution. This is in contrast to most methods for acetal formation which usually employ acid-catalyzed conditions.

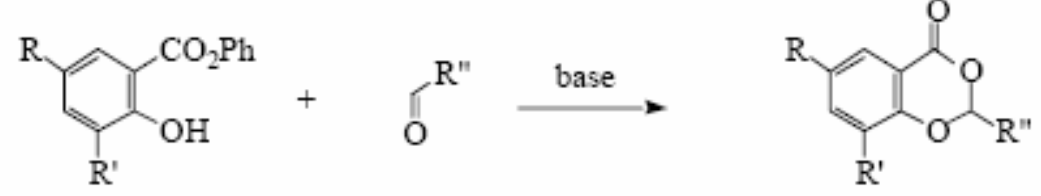

\section{Scheme 1}

Herein we provide further examples of this process as well full experimental details for these new cases as well as the first successful reaction with an aromatic aldehyde.

\section{Results and Discussion}

Oxo-acetal formations of esters 1 to 3 were investigated. The structures of the starting materials and products are shown in Figure 1. Most reactions were carried out neat except where either, or both, the aldehyde and salicylate were solids. In those cases a very small volume of chloroform 
was added to effect solution. The ratio of reactants was salicylate: aldehyde: base $=1: 5: 1$.<smiles>COc1ccc(C(=O)Oc2ccccc2)c(O)c1</smiles>

1
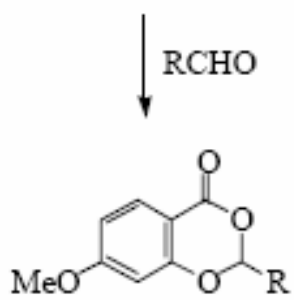

4 a $\mathrm{R}=\mathrm{H}$

b $\mathrm{R}=\mathrm{Me}$

c $\mathrm{R}=\mathrm{Et}$

d $R=i-\operatorname{Pr}$

e $\mathrm{R}=\mathrm{Ph}$<smiles>O=C(O)c1cc2ccccc2cc1O</smiles>

2
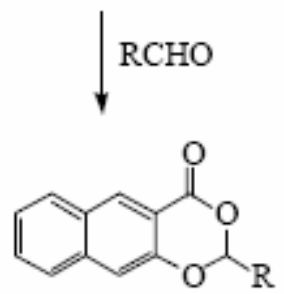

5 a $\mathrm{R}=\mathrm{H}$

b $\mathrm{R}=\mathrm{Me}$

c $\mathrm{R}=\mathrm{Et}$

d $R=i-P r$

e $\mathrm{R}=\mathrm{n}-\mathrm{Bu}$

f $\mathrm{R}=\mathrm{CHCHPh}$<smiles>O=C(Oc1ccccc1)c1ccc2ccccc2c1O</smiles>

3

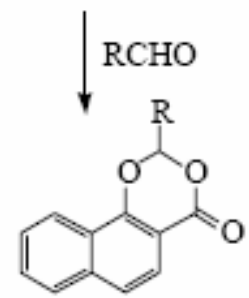

6 a $\mathrm{R}=\mathrm{H}$

b $\mathrm{R}=\mathrm{Et}$

c $\mathrm{R}=\mathrm{i}-\mathrm{Pr}$

d $\mathrm{R}=\mathrm{n}-\mathrm{Bu}$

\section{Figure 1}

As before aliphatic aldehydes, solid paraformaldehyde and chloral all reacted well with esters 1 to 3. In our previous report we noted that the use of aromatic aldehydes did not lead to successful reaction. Interestingly we have now discovered one exception to this, namely reaction of phenyl 4-methoxysalicylate (1). Reaction with benzaldehyde under standard conditions gave oxo-acetal 4e in 49\% yield. Only in one case, that of salicylate 5 was any product formed in a reaction with an unsaturated aldehyde (cinnamaldehyde). The yield was very low (8\%). Hence it can be concluded that, in general, unsaturated aldehydes (including aromatic aldehydes) are not usually good substrates for this process.

A brief investigation was carried out to examine whether any diastereoselectivity would occur in reactions with the chiral, non-racemic aldehyde, D-glyceraldehyde acetonide ${ }^{2}$. Hence 2 was reacted with D-glyceraldehyde acetonide. This provided an essentially 1:1 diastereomeric mixture of acetals 7. Unfortunately this mixture proved to be inseparable.
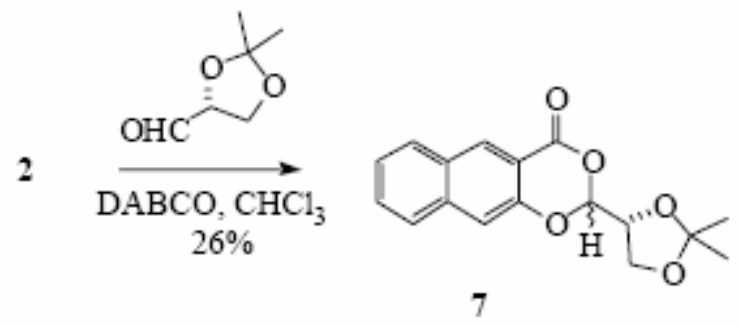

Similar reaction of $\mathbf{3}$ led to another inseparable 1:1 mixture of acetals $\mathbf{8}$. 

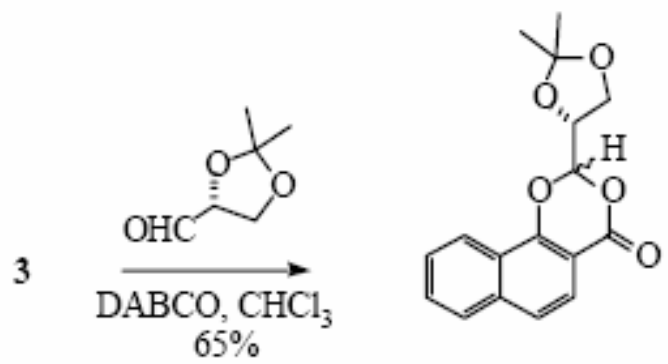

8

In conclusion, this facile process represents one of the very few base-promoted methods for acetal formation. ${ }^{3}$ In this report, we have successfully extended the range of salicylate and salicylate-type esters which undergo this reaction. ${ }^{4}$

\section{Experimental Section}

General Procedures. Melting points were determined on a Kofler hotstage and are uncorrected. Elemental microanalyses were performed by the Australian Microanalytical Service, National Analytical Laboratories, Melbourne and the University of Otago, Dunedin, New Zealand. Optical rotations were recorded on a Perkin Elmer Model 141 Polarimeter. Infrared (IR) spectra were recorded on a Perkin-Elmer 1600 Series Fourier Transform spectrophotometer $\left(\mathrm{cm}^{-1} \mathrm{scale}\right)$ and refer to thin films of liquids (neat) or paraffin (Nujol) mulls of solids between $\mathrm{NaCl}$ plates. High resolution hydrogen-1 nuclear magnetic resonance ( ${ }^{1} \mathrm{H}$ NMR) spectra were recorded at 300 $\mathrm{MHz}$ on a Bruker DPX-300 spectrometer or $400 \mathrm{MHz}$ on a Bruker Avance DRX 400 spectrometer. The ${ }^{1} \mathrm{H}$ NMR spectral data refer to deuteriochloroform solutions $\left(\mathrm{CDCl}_{3}\right)$ using tetramethylsilane (TMS) as internal reference ( $\delta 0.00 \mathrm{ppm})$. Carbon-13 nuclear magnetic resonance $\left({ }^{13} \mathrm{C}\right.$ NMR) spectra were recorded at $75 \mathrm{MHz}$ on a Bruker APX-300 spectrometer or $100 \mathrm{MHz}$ on a Bruker Avance DRX 400 spectrometer. Mass spectrometry (ESI) was performed using samples in methanol on a Micromass Platform QMS Electrospray mass spectrometer. High resolution mass spectra (HRMS) for accurate mass determinations were recorded on a Bruker BioApex 47e FTMS fitted with an Analytica electrospray source using NaI for accurate mass calibration (accuracy $\pm 3 \mathrm{ppm}$ ). Low resolution mass spectra were recorded on a VG micromass 70/70F or a VG TRIO-1 mass spectrometer with an ion source temperature of $200{ }^{\circ} \mathrm{C}$ and electron impact energy of $70 \mathrm{eV}$. Esters 2 and 3 were purchased from Aldrich Chemical Company.

\section{General procedure for preparation of cyclic oxo-acetals}

Unless otherwise indicated, a solution of salicylate ester, aldehyde (5 mol equivalent) and base (1.0 mol equivalent) was stirred at room temperature and allowed to react until TLC analysis indicated complete consumption of starting material. In those cases where one of the starting materials was a solid, and hence the reaction mixture was heterogeneous, a small amount of 
chloroform (usually about $0.2 \mathrm{~mL}$ ) was added to effect dissolution. The reaction mixture was then dissolved in diethyl ether $(20 \mathrm{~mL})$ and the ethereal solution was then washed with water $(3 \times 5 \mathrm{ml})$. The organic layer was dried $\left(\mathrm{MgSO}_{4}\right)$, filtered and concentrated under reduced pressure. Purification of the crude product was carried out either by recrystallization, flash column chromatography or preparative TLC (eluant 1:4, ethyl acetate/ light petroleum). Most reactions were carried out using 500mg of the salicylate ester in each case. In the following only reaction times, chromatography details (eluant, Rf), isolated yield, physical constants and spectroscopic data are given.

2-Hydroxy-4-methoxybenzoic acid $^{5}$. To a solution of (15.4 g, $\left.0.1 \mathrm{~mol}\right)$ of 2,4dihydroxybenzoic acid in $20 \% \mathrm{NaOH}(50 \mathrm{~mL})$ was added $(10.41 \mathrm{~mL}, 0.11 \mathrm{~mol})$ of dimethyl sulfate. The resulting orange solution was stirred at room temperature for 20 hours before being neutralized with concentrated $\mathrm{HCl}$. The mixture was extracted with ether $(3 \times 100 \mathrm{ml})$ and the aqueous layer was acidified to $\mathrm{pH} \sim 1$ with concentrated $\mathrm{HCl}$, resulting in white precipitate. The precipitate was collected by filtration, washed with water and dried (under vacuum). Recrystallization from ethanol and water afforded $(9.7 \mathrm{~g}, 58 \%)$ of product as cream solid. m.p. 145-146 ${ }^{\circ} \mathrm{C}$ (lit. ${ }^{5} 157-159{ }^{\circ} \mathrm{C}$ ). IR (Nujol) 3050-2554b, 1649s, 1624s, 1576m, 1504m, 1465m, $1436 \mathrm{~s}, 1348 \mathrm{~m}, 1360 \mathrm{~m}, 1316 \mathrm{~m}, 1261 \mathrm{~m}, 1250 \mathrm{~m}, 1226 \mathrm{~m}, 1205 \mathrm{~m}, 1176 \mathrm{~m}, 1150 \mathrm{~m}, 1095 \mathrm{~m}, 1025 \mathrm{~m}$, 966m, 962 $\mathrm{m} \mathrm{cm}^{-1} ;{ }^{1} \mathrm{H}$ NMR $\left(200 \mathrm{MHz}, \mathrm{CDCl}_{3}\right) \delta 10.69(\mathrm{~s}, 1 \mathrm{H}, \mathrm{OH}), 7.82(\mathrm{~d}, \mathrm{~J}=9.26 \mathrm{~Hz}, 1 \mathrm{H}$, $\mathrm{H} 5), 6.49(\mathrm{~d}, 1 \mathrm{H}, \mathrm{H} 6), 6.47(\mathrm{~s}, 1 \mathrm{H}, \mathrm{H} 3), 3.85\left(\mathrm{~s}, \mathrm{CH}_{3}\right) ;{ }^{13} \mathrm{C} \mathrm{NMR}\left(50 \mathrm{MHz}, \mathrm{CDCl}_{3}\right) \delta 172.5(\mathrm{CO})$, 166.9 (C-Ar), 165.3 (C-Ar), 132.6 (C-Ar), 107.9 (C5), 106.0 (C6), 101.5 (C3), 55.9 ( $\left.\mathrm{CH}_{3}\right)$.

Phenyl 2-hydroxy-4-methoxybenzoate (1). 2-Hydroxy-4-methoxybenzoic acid (3.00 g, $17.86 \mathrm{mmol})$, phenol $(1.68 \mathrm{~g}, 17.86 \mathrm{mmol})$ and $\mathrm{POCl}_{3}(2.34 \mathrm{ml})$ were heated at $120-130^{\circ} \mathrm{C}$ for 45 minutes. The mixture was dissolved in diethyl ether and then extracted with water. The ether layer was dried over $\mathrm{MgSO}_{4}$ and evaporated in vacuo to gave a red oil. The crude material was purified by flash column chromatography (1:4, ethyl acetate/ light petroleum). Fractions containing material of $\mathrm{Rf}=0.46$ were combined and concentrated to afford the product as white solid (4.28 g, 98\%). m.p. 41-42 ${ }^{\circ}$ IR (Nujol) 2924.5s, 2854.0s, 1686.2m, 1625.0m, 1589.8m, $1459.1 \mathrm{~s}, 1376.6 \mathrm{~s}, 1273.9 \mathrm{~m}, 1254.2 \mathrm{~m}, 1194.2 \mathrm{~m}, 1163.4 \mathrm{~m}, 1128.2 \mathrm{~m}, 1070.7 \mathrm{~m}, 1030.1 \mathrm{~m}, 963.2 \mathrm{~m}$, $856.7 \mathrm{~m}, 836.4 \mathrm{~m}, 773.8 \mathrm{~m}, 744,721.8 \mathrm{~m}, 687.8 \mathrm{~m} \mathrm{~cm}^{-1} ;{ }^{1} \mathrm{H}$ NMR $\left(200 \mathrm{MHz}, \mathrm{CDCl}_{3}\right) \delta 10.73(\mathrm{~s}$, 1H, OH), 7.96 (d, J=0.84 Hz, 1H, H5), 7.23 (m, 5H, Ph), 6.55 (d, J=8.45 Hz, 1H, H6), 6.51 (s, 1H, H3), 3.87 (s, 3H, CH 3$) ;{ }^{13} \mathrm{C}$ NMR $\left(50 \mathrm{MHz}, \mathrm{CDCl}_{3}\right) \delta 168.7$ (CO), 166.2 (C-Ar), 164.4 (CAr), 150.1 (C-Ar), 131.7 (C5), 129.5 (2xCH-Ph), 126.2 (C6), 121.7 (2xCH-Ph), 108.0 (C3), 104.8 (C-Ph), $100.8(\mathrm{CH}-\mathrm{Ph}), 55.6\left(\mathrm{CH}_{3}\right)$; MS m/z 245.14 (M+H) ${ }^{+}$.

7-Methoxy-4-oxo-1,3-benzodioxane (4a). $6 \mathrm{~d}$, $(\mathrm{Rf}=0.25), 79 \%$, colourless crystals (diethyl ether), m.p. $67-68{ }^{\circ} \mathrm{C}$. IR (Nujol) 2922.8s, 2853.6s, 2358.4m, 1742.3m, 1624.6m, 1458.5s, $1376.8 \mathrm{~m}, 1254.8 \mathrm{~m}, 1180.0 \mathrm{w}, 1110.6 \mathrm{w}, 1063.6 \mathrm{w}, 1014.4 \mathrm{w}, 982.1 \mathrm{w}, 929.0 \mathrm{w}, 832.5 \mathrm{w}, 739.7 \mathrm{w}$, 668.0w, 667.9 w cm ${ }^{-1}$; ${ }^{1} \mathrm{H}$ NMR $\left(200 \mathrm{MHz}, \mathrm{CDCl}_{3}\right) \delta 7.9(\mathrm{~d}, \mathrm{~J}=8.87 \mathrm{~Hz}, 1 \mathrm{H}, \mathrm{H} 5), 6.73$ (dd, J=6.42 $\mathrm{Hz}, 1 \mathrm{H}, \mathrm{H} 6), 6.51(\mathrm{~s}, 1 \mathrm{H}, \mathrm{H} 8), 5.63\left(\mathrm{~s}, 2 \mathrm{H}, \mathrm{CH}_{2}\right), 3.86\left(\mathrm{~s}, 3 \mathrm{H}, \mathrm{CH}_{3} \mathrm{O}\right) ;{ }^{13} \mathrm{C} \mathrm{NMR}(50 \mathrm{MHz}$, $\mathrm{CDCl}_{3}$ ) $\delta 166.0$ (CO), 161.3 (C-Ar), 160.2 (C-Ar), 131.8 (C5), 111.4 (C6), 107.3 (C-Ar), 100.3 
(C8), $91.0(\mathrm{C} 2), 55.7\left(\mathrm{CH}_{3} \mathrm{O}\right)$; HRMS calcd. for $\mathrm{C}_{9} \mathrm{H}_{8} \mathrm{O}_{4}: \mathrm{m} / \mathrm{z}$ 180.0423, found 180.0421; MS m/z $180(58), 150$ (100), 122 (66).

2-Methyl-7-methoxy 4-oxo-1,3-benzodioxane (4b). $2 \mathrm{~d},(\mathrm{Rf}=0.16)(94 \%)$, white crystals (diethyl ether), m.p. 59-60 ${ }^{\circ} \mathrm{C}$. IR (Nujol) 3436.0b, 2923.7s, 2853.5s, $1741.1 \mathrm{~m}, 1616.4 \mathrm{~m}$, $1460.1 \mathrm{~s}, 1376.9 \mathrm{~s}, 1263.0 \mathrm{~m}, 1094.0 \mathrm{~m}, 1025.6 \mathrm{~m}, 929.4 \mathrm{w}, 847.9 \mathrm{w}, 767.9 \mathrm{w}, 734.4 \mathrm{w}, 688.2 \mathrm{w} \mathrm{cm}{ }^{-1}$; ${ }^{1} \mathrm{H}$ NMR (200 MHz, $\left.\mathrm{CDCl}_{3}\right) \delta 7.89(\mathrm{~d}, \mathrm{~J}=8.87 \mathrm{~Hz}, 1 \mathrm{H}, \mathrm{H} 5), 6.71$ (dd, J=2.39, $6.44 \mathrm{~Hz}, 1 \mathrm{H}, \mathrm{H6}$ ), 6.48 (d, J=2.38 Hz, 1H, H8), 5.73 (q, J=5.17 Hz, 1H, H2), $3.86\left(\mathrm{~s}, 3 \mathrm{H}, \mathrm{CH}_{3} \mathrm{O}\right), 1.73$ (d, J=5.17 $\left.\mathrm{Hz}, 3 \mathrm{H}, \mathrm{CH}_{3}\right) ;{ }^{13} \mathrm{C} \mathrm{NMR}\left(50 \mathrm{MHz}, \mathrm{CDCl}_{3}\right) \delta 166.1$ (CO), 162.2 (C-Ar), 160.3 (C-Ar), 131.7 (C5), 111.2 (C6), 106.9 (C-Ar), 100.2 (C8), 98.9 (C2), 55.8 ( $\left.\mathrm{CH}_{3} \mathrm{O}\right), 19.9$ (CH3); HRMS calc'd for $\mathrm{C}_{10} \mathrm{H}_{10} \mathrm{O}_{4}: \mathrm{m} / \mathrm{z}$ 194.0579, found 194.0562; MS m/z 195.06 (M+H) ${ }^{+}$.

2-Ethyl-7-methoxy 4-oxo-1,3-benzodioxane (4c). 28 h, ( $\mathrm{Rf}=0.25),(86 \%)$, yellow oil. IR (neat) $3458.6 \mathrm{w}, 3079.7 \mathrm{w}, 2979.0 \mathrm{~s}, 2942.1 \mathrm{~s}, 2844.1 \mathrm{~m}, 2364.1 \mathrm{w}, 2017.7 \mathrm{w}, 1744.1 \mathrm{~s}, 1614.7 \mathrm{~s}, 1584.9 \mathrm{~s}$, $1501.0 \mathrm{~s}, 1451.3 \mathrm{~s}, 1395.1 \mathrm{~s}, 1332.1 \mathrm{~m}, 1258.8 \mathrm{~s}, 1201.6 \mathrm{~s}, 1169.9 \mathrm{~s}, 1133.9 \mathrm{~s}, 1088.6 \mathrm{~s}, 1050.7 \mathrm{~s}$, $1022.9 \mathrm{~s}, 985.8 \mathrm{w}, 964.1 \mathrm{~s}, 930.5 \mathrm{~m}, 838.6 \mathrm{~s}, 800.4 \mathrm{w}, 769.9 \mathrm{~s}, 690.0 \mathrm{~s}, 665.2 \mathrm{w}, 649.3 \mathrm{~m}, 611.6 \mathrm{w}$, $573.1 \mathrm{~s} \mathrm{~cm}^{-1}$; ${ }^{1} \mathrm{H}$ NMR (200 MHz, $\left.\mathrm{CDCl}_{3}\right) \delta 7.9(\mathrm{~d}, \mathrm{~J}=8.83 \mathrm{~Hz}, 1 \mathrm{H}, \mathrm{H} 5), 6.71$ (dd, J=2.39, 6.33 $\mathrm{Hz}, 1 \mathrm{H}, \mathrm{H} 6), 6.50$ (d, J=2.37 Hz, 1H, H8), 5.56 (m, 1H, H2), 3.87 (s, 3H, $\left.\mathrm{CH}_{3} \mathrm{O}\right), 2.04$ (m, 2H, $\left.\mathrm{CH}_{2}\right), 1.4\left(\mathrm{t}, \mathrm{J}=7.46,7.54 \mathrm{~Hz}, 3 \mathrm{H}, \mathrm{CH}_{3}\right) ;{ }^{13} \mathrm{C} \mathrm{NMR}\left(50 \mathrm{MHz}, \mathrm{CDCl}_{3}\right) \delta 165.9(\mathrm{CO}), 162.1(\mathrm{C}-\mathrm{Ar})$, 160.1 (C-Ar), 131.4 (C5), 110.9 (C6), 106.7 (C-Ar), 101.9 (C8), 100.0 (C2), $55.6\left(\mathrm{CH}_{3} \mathrm{O}\right), 26.6$ $\left(\mathrm{CH}_{2}\right), 6.9\left(\mathrm{CH}_{3}\right)$; HRMS calc'd for $\mathrm{C}_{11} \mathrm{H}_{12} \mathrm{O}_{4}: \mathrm{m} / \mathrm{z}$ 208.0736, found 208.0739; MS m/z 209.09 $(\mathrm{M}+\mathrm{H})^{+}$.

2-Isopropyl-7-methoxy 4-oxo-1,3-benzodioxane (4d). $4 \mathrm{~d},(\mathrm{Rf}=0.27)$, (87\%), yellow oil. IR (neat) $3462.2 \mathrm{w}, 2972.3 \mathrm{~m}, 2844.0 \mathrm{~m}, 2362.3 \mathrm{~m}, 1742.8 \mathrm{~s}, 1618.3 \mathrm{~s}, 1584.9 \mathrm{~s}, 1500.3 \mathrm{~s}, 1448.8 \mathrm{~s}$, $1397.2 \mathrm{~s}, 1375.6 \mathrm{~s}, 1332.3 \mathrm{~m}, 1261.3 \mathrm{~s}, 1201.7 \mathrm{~s}, 1167.2 \mathrm{~s}, 1110.5 \mathrm{~s}, 1079.3 \mathrm{~m}, 1048.3 \mathrm{~m}, 1020.4 \mathrm{~m}$, $982.6 \mathrm{~m}, 959.5 \mathrm{~m}, 837.8 \mathrm{~m}, 768.4 \mathrm{~m}, 691.9 \mathrm{~m}, 699.9 \mathrm{w}, 652.2 \mathrm{w}, 617.6 \mathrm{w} \mathrm{cm}{ }^{-1}$; ${ }^{1} \mathrm{H}$ NMR $(200 \mathrm{MHz}$, $\left.\mathrm{CDCl}_{3}\right) \delta 7.89(\mathrm{~d}, \mathrm{~J}=8.81 \mathrm{~Hz}, 1 \mathrm{H}, \mathrm{H} 5), 6.72(\mathrm{dd}, \mathrm{J}=2.39 \mathrm{~Hz}, 1 \mathrm{H}, \mathrm{H6}), 6.67$ (d, J=2.39 Hz, 1H, $\mathrm{H} 8), 5.36$ (d, J=4.22 Hz, 1H, H2), 3.86 (s, 3H, CH $3 \mathrm{O}), 2.39$ (m, 1H, CH), 1.14 (d, J=6.89 Hz, 6H, $\left.2 \mathrm{xCH}_{3}\right) ;{ }^{13} \mathrm{C} \mathrm{NMR}\left(50 \mathrm{MHz}, \mathrm{CDCl}_{3}\right) \delta 165.9(\mathrm{CO}), 162.3$ (C-Ar), 160.3 (C-Ar), 131.4 (C5), 111.0 (C6), 106.8 (C-Ar), $104.4(\mathrm{C} 8), 100.1(\mathrm{C} 2), 55.6\left(\mathrm{CH}_{3} \mathrm{O}\right), 31.6\left(\mathrm{C}-\mathrm{i}\right.$ Pr), $15.9\left(2 \mathrm{xCH}_{3}\right)$; HRMS calc. for $\mathrm{C}_{12} \mathrm{H}_{14} \mathrm{O}_{4}: \mathrm{m} / \mathrm{z} 222.0892$, found 222.0897; MS m/z 223.10 (M+H) ${ }^{+}$.

2-Phenyl-7-methoxy 4-oxo-1,3-benzodioxane (4e). $5 \mathrm{~d}, 60^{\circ} \mathrm{C},(\mathrm{Rf}=0.24),(49 \%)$, brown solid, m.p. $75-76^{\circ} \mathrm{C}$. IR (Nujol) 2920.5s, 2725.8w, 1759.8m, 1726.0m, 161803m, 1582.2m, 1463.0s, $1376.9 \mathrm{~s}, 1315.0 \mathrm{~m}, 1260.4 \mathrm{~m}, 1197.7 \mathrm{~m}, 1116.5 \mathrm{~m}, 1074.1 \mathrm{~m}, 1016.1 \mathrm{~m}, 944.8 \mathrm{~m}, 889.1 \mathrm{~m}, 833.7 \mathrm{~m}$, 794.7w, 722.4m, 697.0w, 668.0w cm ${ }^{-1} ;{ }^{1} \mathrm{H}$ NMR (200 MHz, $\left.\mathrm{CDCl}_{3}\right) \delta 7.94(\mathrm{~d}, \mathrm{~J}=8.82 \mathrm{~Hz}, 1 \mathrm{H}$, H5), 7.64-7.47 (m, 5H, Ph), 6.74 (dd, J=2.39, 6.37 Hz, 1H, H6), 6.56 (d, J=2.37 Hz, 1H, H8), $6.51(\mathrm{~s}, 1 \mathrm{H}, \mathrm{H} 2), 3.85$ (s, 3H, $\left.\mathrm{CH}_{3} \mathrm{O}\right) ;{ }^{13} \mathrm{C} \mathrm{NMR}\left(300 \mathrm{MHz}, \mathrm{CDCl}_{3}\right) \delta 166.1(\mathrm{CO}), 161.65(\mathrm{C}-\mathrm{Ar})$, 159.9 (C-Ar), 134.1 (C-Ph), 131.7 (C5), 130.2 (C6), 128.5 (2xCH-Ph), 126.5 (2xCH-Ph), 111.5 (CH-Ph), 107.0 (C-Ar), 100.6 (C8), 100.4 (C2), $55.9\left(\mathrm{CH}_{3} \mathrm{O}\right)$; HRMS calc. for $\mathrm{C}_{15} \mathrm{H}_{12} \mathrm{O}_{4}: \mathrm{m} / \mathrm{z}$ 256.0736, found 256.0739; MS m/z $262(\mathrm{M}+\mathrm{Na})^{+}$.

4-Oxo-1,3-(b)-naphthodioxane (5a). $2 \mathrm{~d},(\mathrm{Rf}=0.84),(44 \%)$, white crystals (diethyl ether), m.p. 105-106 ${ }^{\circ} \mathrm{C}$. IR (Nujol) 2922.1s, 2852.8s, 1749.7m, 1638.4w, 1608.5w, 1560.2w, 1460.2s, 
$1376.9 \mathrm{~s}, 1345.3 \mathrm{~m}, 1277.6 \mathrm{w}, 1257.4 \mathrm{~m}, 1205.4 \mathrm{~m}, 1155.9 \mathrm{w}, 1124.4 \mathrm{w}, 1042.8 \mathrm{w}, 1019.7 \mathrm{w}, 995.0 \mathrm{~m}$, 951.6m, 915.7w, 881.8m, 853.9m, 773.0m, 738.1m, 722.2 $\mathrm{m} \mathrm{cm}^{-1} ;{ }^{1} \mathrm{H} \mathrm{NMR}\left(300 \mathrm{MHz}, \mathrm{CDCl}_{3}\right)$ $\delta 8.66$ (s, 1H, H5), 7.95 (d, J=8.28 Hz, 1H, H6), 7.81 (d, J=8.32 Hz, 1H, H9), 7.62 (t, J=1.17, 1.27 $\mathrm{Hz}, 1 \mathrm{H}, \mathrm{H} 7), 7.48$ (t, J=1.14 Hz, 1H, H8), 7.46 (s, 1H, H10), $5.74\left(\mathrm{~s}, 2 \mathrm{H}, \mathrm{CH}_{2}\right) ;{ }^{13} \mathrm{C}$ NMR $(50$ $\mathrm{MHz}, \mathrm{CDCl}_{3}$ ) $\delta 161.7$ (CO), 153.3 (C-Ar), 137.3 (C-Ar), 133.0 (C5), 129.8 (C6), 129.6 (C9), 129.4 (C-Ar), 127.0 (C7), 125.7 (C8), 115.1 (C-Ar), 112.3 (C10), 91.4 (C2); MS m/z 200 (51), 170 (100), 142 (89), 114 (74); Anal. Calc. for $\mathrm{C}_{12} \mathrm{H}_{8} \mathrm{O}_{3}$ : C, 71.99. H, 4.03. Found: C, 71.60; H, 3.81 .

2-Methyl 4-oxo-1,3-(b)-naphthodioxane (5b). $2 \mathrm{~d}, \mathrm{rt}, \mathrm{K}_{2} \mathrm{CO}_{3}$ in place of DABCO, $(\mathrm{Rf}=0.27)$, (14\%), white solid, m.p. 90-91 ${ }^{\circ} \mathrm{C}$. IR (Nujol) 2923.1s, 2854.1s, $1726.4 \mathrm{~m}, 1636.1 \mathrm{~m}, 1608.1 \mathrm{~m}$, $1504.4 \mathrm{~m}, 1463.7 \mathrm{~s}, 1270.6 \mathrm{~s}, 1255.0 \mathrm{~s}, 1215.3 \mathrm{~s}, 1135.9 \mathrm{~m}, 1084.9 \mathrm{~m}, 1029.4 \mathrm{~s}, 934.0 \mathrm{~s}, 881.8 \mathrm{~s}$, $781.7 \mathrm{~s}, 754.1 \mathrm{~s}, 720.2 \mathrm{~m} \mathrm{~cm}^{-1} ;{ }^{1} \mathrm{H}$ NMR $\left(300 \mathrm{MHz} \mathrm{CDCl}_{3}\right) \delta 8.63(\mathrm{~s}, 1 \mathrm{H}, \mathrm{H} 5), 7.92$ (d, J=8.3 Hz, 1H, H6), 7.78 (d, J=8.3 Hz, 1H, H9), 7.59 (t, J=7.50 Hz, 1H, H7), 7.46 (t, J=7.60 Hz, 1H, H8), $7.40(\mathrm{~s}, 1 \mathrm{H}, \mathrm{H} 10), 5.8(\mathrm{~m}, 1 \mathrm{H}, \mathrm{H} 2), 1.8\left(\mathrm{~d}, \mathrm{~J}=5.2 \mathrm{~Hz}, 3 \mathrm{H}, \mathrm{CH}_{3}\right) ;{ }^{13} \mathrm{C} \mathrm{NMR}\left(50 \mathrm{MHz}, \mathrm{CDCl}_{3}\right)$

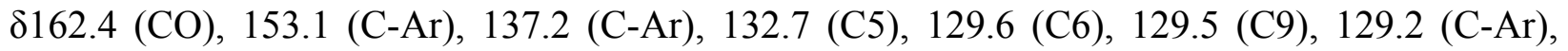
127.0 (C7), 125.5 (C8), 114.5 (C-Ar), 112.0 (C10), 99.2 (C2), $20.1\left(\mathrm{CH}_{3}\right) ; \mathrm{MS} \mathrm{m} / \mathrm{z} 214$ (100), 171 (50), 170 (77), 143 (47), 142 (84), 115 (39), 114 (67), 113 (74), 88 (46), 87 (27), 86 (19), 76 (11), 75 (77), 74 (23), 71 (24), 63 (49), 62 (29), 57 (13).

2-Ethyl 4-oxo-[1,3-b]-naphthodioxane (5c). $2 \mathrm{~d},(\mathrm{Rf}=0.35),(75 \%)$, colourless crystals (diethyl ether), m.p. $81-82{ }^{\circ} \mathrm{C}$. IR (Nujol) 2851.1s, 2725.4w, 1762.2w, 1728.1m, 1637.4m, 1607.2w, $1463.1 \mathrm{~s}, 1377.1 \mathrm{~s}, 1310.2 \mathrm{w}, 1271.1 \mathrm{w}, 1244.4 \mathrm{~m}, 1207.1 \mathrm{~m}, 1148.6 \mathrm{w}, 1033.0 \mathrm{w}, 947.8 \mathrm{~m}, 896.4 \mathrm{w}$, 873.3w, 846.7w, 817.7w, 779.3w, 722.2 $\mathrm{m} \mathrm{cm}^{-1}$; ${ }^{1} \mathrm{H}$ NMR (300 MHz, $\left.\mathrm{CDCl}_{3}\right) \delta 8.63$ (s, 1H, H5), 7.93 (d, J=8.34 Hz, 1H, H6), 7.78 (d, J=8.34 Hz, 1H, H9), 7.59 (t, J=1.21 Hz, 1H, H7), 7.46 (t, $\mathrm{J}=1.15,1.09 \mathrm{~Hz}, 1 \mathrm{H}, \mathrm{H} 8), 7.42$ (s, 1H, H10), 5.64 (t, J=4.92 Hz, 1H, CH), 2.12 (m, J=4.94 Hz, $\left.2 \mathrm{H}, \mathrm{CH}_{2}\right), 1.18\left(\mathrm{t}, \mathrm{J}=7.49,7.60 \mathrm{~Hz}, 3 \mathrm{H}, \mathrm{CH}_{3}\right) ;{ }^{13} \mathrm{C} \mathrm{NMR}\left(50 \mathrm{MHz}, \mathrm{CDCl}_{3}\right) \delta 162.6(\mathrm{CO}), 153.3$ (C-Ar), 137.3 (C-Ar), 132.7 (C5), 129.6 (C6), 129.5 (C9), 129.3 (C-Ar), 127.0 (C7), 125.5 (C8), 114.7 (C-Ar), 112.1 (C10), $102.5(\mathrm{C} 2), 27.1\left(\mathrm{CH}_{2}\right), 7.2\left(\mathrm{CH}_{3}\right)$; MS m/z 228 (18), $170(100), 142$ (54), 114 (53), 113 (20); Anal. Calc. for $\mathrm{C}_{14} \mathrm{H}_{12} \mathrm{O}_{3}$ : C, 73.60. H, 5.30. Found: C, 73.13; H, 5.23.

2-Isopropyl 4-oxo-[1,3-b]-naphthodioxane (5d). $3 \mathrm{~d},(\mathrm{Rf}=0.42),(87 \%)$, white crystals (diethyl ether), m.p. $58-59{ }^{\circ} \mathrm{C}$. IR (Nujol) 2923.9s, 2854.0s, 2280.6w, 1760.5m, 1639.5m, 1609.4w, $1460.3 \mathrm{~s}, 1377.1 \mathrm{~s}, 1344.3 \mathrm{~m}, 1296.9 \mathrm{~m}, 1268.2 \mathrm{~m}, 1245.5 \mathrm{~m}, 1209.6 \mathrm{~m}, 1135.8 \mathrm{w}, 1062.1 \mathrm{~m}, 986.3 \mathrm{~m}$, 961.5m, 917.4m, 875.9m, 831.0w, 807.5w, 776.4m, 745.9m, 722.6m cm ${ }^{-1}$; ${ }^{1} \mathrm{H} \mathrm{NMR} \mathrm{(300} \mathrm{MHz,}$ $\left.\mathrm{CDCl}_{3}\right) \delta 8.63(\mathrm{~s}, 1 \mathrm{H}, \mathrm{H} 5), 7.92$ (d, J=8.32 Hz, 1H, H6), 7.78 (d, J=8.39 Hz, 1H, H9), 7.59 (t, $\mathrm{J}=1.24,1.30 \mathrm{~Hz}, 1 \mathrm{H}, \mathrm{H} 7), 7.46$ (t, J=1.22, $6.78 \mathrm{~Hz}, 1 \mathrm{H}, \mathrm{H} 8), 7.42$ (s, 1H, H10), 5.45 (d, J=4.30 $\mathrm{Hz}, 1 \mathrm{H}, \mathrm{H} 2), 2.29(\mathrm{~m}, 1 \mathrm{H}, \mathrm{CH}), 1.18\left(\mathrm{~d}, \mathrm{~J}=6.92 \mathrm{~Hz}, 6 \mathrm{H}, 2 \mathrm{xCH}_{3}\right) ;{ }^{13} \mathrm{C} \mathrm{NMR}\left(50 \mathrm{MHz}, \mathrm{CDCl}_{3}\right) \delta$ 162.8 (CO), 153.4 (C-Ar), 137.4 (C-Ar), 132.7 (C5), 129.6 (C6\&C9), 129.3 (C-Ar), 127.0 (C7), 125.5 (C8), 114.8 (C-Ar), 112.2 (C10), $104.9(\mathrm{C} 2), 31.9(\mathrm{CH}), 16.1\left(2 \mathrm{xCH}_{3}\right)$; MS m/z $242(12)$, 171 (16), 170 (100), 142 (48), 114 (24); Anal. Calc. for $\mathrm{C}_{15} \mathrm{H}_{14} \mathrm{O}_{3}$ : C, 74.36. H, 5.82. Found: C, $74.42 ; \mathrm{H}, 5.64$.

2-Butyl 4-oxo-[1,3-b]-naphthodioxane (5e). $3 \mathrm{~d}$, $(\mathrm{Rf}=0.39),(83 \%)$, colourless crystals (diethyl 
ether), m.p. $85-86{ }^{\circ} \mathrm{C}$. IR (Nujol) 2850.8s, 2724.7w, 1762.6m, 1744.7w, $1637.4 \mathrm{~m}, 1608.6 \mathrm{w}$, $1462.8 \mathrm{~s}, 1377.2 \mathrm{~s}, 1270.9 \mathrm{~m}, 1249.8 \mathrm{~m}, 1202.0 \mathrm{~m}, 1137.8 \mathrm{w}, 1087.5 \mathrm{w}, 1004.4 \mathrm{w}, 970.9 \mathrm{~m}, 914.0 \mathrm{w}$, $791.2 \mathrm{w}, 770.2 \mathrm{~m}, 722.2 \mathrm{~s} \mathrm{~cm}^{-1} ;{ }^{1} \mathrm{H}$ NMR $\left(200 \mathrm{MHz}, \mathrm{CDCl}_{3}\right) \delta 8.63$ (s, 1H, H5), 7.93 (d, J=7.59 Hz, 1H, H6), 7.79 (d, J=7.59 Hz, 1H, H9), 7.62 (t, J=1.28, $1.30 \mathrm{~Hz}, 1 \mathrm{H}, \mathrm{H} 7), 7.46$ (d, J=5.50 Hz, $1 \mathrm{H}, \mathrm{H} 8), 7.41$ (s, 1H, H10), $5.68(\mathrm{t}, \mathrm{J}=5.07,5.01 \mathrm{~Hz}, 1 \mathrm{H}, \mathrm{H} 2), 2.09\left(\mathrm{~m}, 2 \mathrm{H}, \mathrm{CH}_{2}\right), 1.60(\mathrm{~m}, 2 \mathrm{H}$, $\left.\mathrm{CH}_{2}\right), 1.43\left(\mathrm{~m}, 2 \mathrm{H}, \mathrm{CH}_{2}\right), 0.99\left(\mathrm{t}, \mathrm{J}=7.08 \mathrm{~Hz}, 3 \mathrm{H}, \mathrm{CH}_{3}\right) ;{ }^{13} \mathrm{C} \mathrm{NMR}\left(50 \mathrm{MHz}, \mathrm{CDCl}_{3}\right) \delta 162.5$ (CO), 153.2 (C-Ar), 137.2 (C-Ar), 132.6 (C5), 129.6 (C6), 129.5 (C9), 126.9 (C7), 125.4 (C8), 114.7 (C-Ar), 112.1 (C10), $102.5(\mathrm{C} 2), 33.4\left(\mathrm{CH}_{2}\right), 24.9\left(\mathrm{CH}_{2}\right), 22.2\left(\mathrm{CH}_{2}\right), 13.8\left(\mathrm{CH}_{3}\right) ; \mathrm{MS} \mathrm{m} / \mathrm{z}$ 256 (14), 171 (17), 170 (100), 142 (56), 114 (50), 115 (20), 113 (15); Anal. Calc. for $\mathrm{C}_{16} \mathrm{H}_{16} \mathrm{O}_{3}$ : C, 74.98. H, 6.29. Found: C, 73.79; H, 6.97.

2-Trichloromethyl 4-oxo-[1,3-b]-naphthodioxane (62f). $0.5 \mathrm{~h},(\mathrm{Rf}=0.30),(45 \%)$, pale yellow crystals (diethyl ether), m.p. 179-180 ${ }^{\circ} \mathrm{C}$. IR (Nujol) 3433.3b, 2921.5s, 1753.6m, 1632.1m, $1609.0 \mathrm{w}, 1462.6 \mathrm{~s}, 1377.1 \mathrm{~s}, 1341.5 \mathrm{~m}, 1270.0 \mathrm{w}, 1241.4 \mathrm{~m}, 1203.9 \mathrm{~m}, 1141.5 \mathrm{~m}, 1099.4 \mathrm{w}$, $1059.8 \mathrm{w}, 1015.8 \mathrm{~m}, 910.5 \mathrm{w}, 882.2 \mathrm{w}, 864.4 \mathrm{w}, 835.4 \mathrm{~m}, 765.9 \mathrm{~m}, 722.2 \mathrm{~m} \mathrm{~cm}^{-1} ;{ }^{1} \mathrm{H}$ NMR $(300$ $\mathrm{MHz}_{\mathrm{CDCl}}$ ) $\delta 8.66(\mathrm{~s}, 1 \mathrm{H}, \mathrm{H} 5), 7.96(\mathrm{~d}, \mathrm{~J}=8.34 \mathrm{~Hz}, 1 \mathrm{H}, \mathrm{H} 6), 7.83$ (d, J=8.25 Hz, 1H, H9), 7.65 (t, J=6.97, $7.07 \mathrm{~Hz}, 1 \mathrm{H}, \mathrm{H} 7), 7.58(\mathrm{~s}, 1 \mathrm{H}, \mathrm{H} 10), 7.52$ (t, J=8.10, $7.02 \mathrm{~Hz}, 1 \mathrm{H}, \mathrm{H} 8), 5.95$ (s, 1H, $\mathrm{H} 2) ;{ }^{13} \mathrm{C} \mathrm{NMR}\left(50 \mathrm{MHz}, \mathrm{CDCl}_{3}\right) \delta 159.2$ (CO), 150.9 (C-Ar), 137.5 (C-Ar), 133.1 (C5), 130.3 (C6), 129.7 (C9), 129.7 (C-Ar), 127.2 (C7), 126.3 (C8), 113.3 (C-Ar), 112.9 (C10), 100.9 (C2), $94.4\left(\mathrm{CCl}_{3}\right)$; MS m/z 316 (9), 200 (9), 199 (65), 171 (33), 170 (100), 142 (74), 115 (37), 114 (81), 113 (35), 88 (22), 86 (11), 63 (23); Anal. Calc. for $\mathrm{C}_{13} \mathrm{H}_{7} \mathrm{O}_{3} \mathrm{Cl}_{3}$ : C, 49.17. H, 2.22. Found: C, 49.38; H, 2.21 .

2-Cinnamal 4-oxo-[1,3-b]-naphthodioxane (5g). $3 \mathrm{~d},(\mathrm{Rf}=0.32),(8 \%)$, white needles (diethyl ether), m.p. 163-164 ${ }^{\circ} \mathrm{C}$. IR (Nujol) $3329.6 \mathrm{w}, 2926.0 \mathrm{~s}, 1729.7 \mathrm{~m}, 1711.8 \mathrm{~m}, 1693.9 \mathrm{~m}, 1668.9 \mathrm{~m}$, $1637.8 \mathrm{~m}, 1536.4 \mathrm{~m}, 1463.0 \mathrm{~s}, 1377.1 \mathrm{~s}, 1254.6 \mathrm{~m}, 1207.5 \mathrm{w}, 1144.6 \mathrm{w}, 1050.0 \mathrm{w}, 1018.3 \mathrm{w}, 979.6 \mathrm{~m}$, 953.4m, 879.4w, 772.6w, 722.3m, 693.6w cm ${ }^{-1}$; ${ }^{1} \mathrm{H}$ NMR (300 MHz, $\left.\mathrm{CDCl}_{3}\right) \delta 8.67$ (s, 1H, H5), 7.94 (d, J=8.36 Hz, 1H, Ha), 7.80 (d, J=7.69 Hz, 1H, H9), 7.60 (t, J=1.30, 7.03 Hz, 1H, H7), 7.49 (m, 5H, Ph), 7.37 (t, J=1.64, $1.69 \mathrm{~Hz}, 1 \mathrm{H}, \mathrm{H} 8), 7.35$ (s, 1H, H10), 7.06 (d, J=15.95 Hz, 1H, H6), $6.45(\mathrm{dd}, \mathrm{J}=5.24,37.92,5.13 \mathrm{~Hz}, 1 \mathrm{H}, \mathrm{Hb}), 6.27(\mathrm{~d}, \mathrm{~J}=1.16 \mathrm{~Hz}, 1 \mathrm{H}, \mathrm{H} 2) ;{ }^{13} \mathrm{C} \mathrm{NMR}(50 \mathrm{MHz}$, $\mathrm{CDCl}_{3}$ ) $\delta 162.2(\mathrm{CO}), 152.9$ (C-Ar), 137.5 (C-Ar), 136.8 (Ca), 134.9 (C-Ar), 132.9 (C5), 129.8 (C6), 129.7 (C9), 129.4 (C-Ar), 129.2 (CH-Ph), 128.8 (2xCH-Ph), 127.2 (2xCH-Ph), 127.1 (C7), 125.7 (C8), 121.2 (Cb), 114.9 (C-Ar), 112.6 (C10), 100.4 (C2); HRMS calc. for $\mathrm{C}_{20} \mathrm{H}_{14} \mathrm{O}_{3}: \mathrm{m} / \mathrm{z}$ 302.0943, found 302.0948; MS m/z $303.1(\mathrm{M}+\mathrm{H})^{+}$.

2-Isopropylidene glyceral 4-oxo-[1,3-b]-naphthodioxane (7). $3 \mathrm{~d},(\mathrm{Rf}=0.80),(26 \%)$, colorless crystals (diethyl ether), m.p. 163-164 ${ }^{\circ} \mathrm{C},[\alpha]_{\mathrm{D}}{ }^{0} 14.192$ (c 2.5, $\mathrm{CHCl}_{3}$ ). IR (Nujol) 2925.3s, $2280.8 \mathrm{w}, 1749.1 \mathrm{~m}, 1637.1 \mathrm{~m}, 1462.6 \mathrm{~s}, 1377.1 \mathrm{~s}, 1347.1 \mathrm{~m}, 1303.8 \mathrm{~m}, 1252.1 \mathrm{w}, 1210.6 \mathrm{~m}$, $1151.4 \mathrm{w}, 1080.9 \mathrm{w}, 1019.6 \mathrm{~m}, 889.6 \mathrm{w}, 843.1 \mathrm{w}, 775.9 \mathrm{w}, 722.0 \mathrm{~m} \mathrm{~cm}^{-1}$; ${ }^{1} \mathrm{H} \mathrm{NMR}(300 \mathrm{MHz}$, $\left.\mathrm{CDCl}_{3}\right) \delta 8.63(\mathrm{~s}, 1 \mathrm{H}, \mathrm{H} 5), 7.93$ (d, J=8.28 Hz, 1H, H6), 7.78 (d, J=8.28 Hz, 1H, H9), 7.61 (t, $\mathrm{J}=1.16 \mathrm{~Hz}, 1 \mathrm{H}, \mathrm{H} 7), 7.48$ (t, J=7.07 Hz, 1H, H8), 7.44 (s, 1H, H10), 5.69 (d, J=4.35 Hz, 1H, H2), $4.52(\mathrm{~m}, 1 \mathrm{H}, \mathrm{CH}), 4.29\left(\mathrm{~m}, 2 \mathrm{H}, \mathrm{CH}_{2}\right), 1.50\left(\mathrm{~d}, \mathrm{~J}=33.80 \mathrm{~Hz}, 6 \mathrm{H}, 2 \mathrm{xCH}_{3}\right) ;{ }^{13} \mathrm{C} \mathrm{NMR}(50 \mathrm{MHz}$, $\mathrm{CDCl}_{3}$ ) $\delta 161.3$ (CO), 152.5 (C-Ar), 137.3 (C-Ar), 132.9 (C5), 129.9 (C6), 129.7 (C9), 129.5 (C- 
Ar), 127.1 (C7), 125.8 (C8), 114.6 (C-Ar), 112.5 (C10), 110.9 (C $\left.{ }^{\mathrm{i}} \mathrm{Pr}\right), 99.8$ (C2), 75.02 (CH), 64.6 $\left(\mathrm{CH}_{2}\right), 26.3\left(\mathrm{CH}_{3}\right), 25.5\left(\mathrm{CH}_{3}\right)$; MS m/z $301.2(\mathrm{M}+\mathrm{H})^{+}$; Anal. Calc. for $\mathrm{C}_{17} \mathrm{H}_{16} \mathrm{O}_{5}: \mathrm{C}, 67.99$. H, 5.37. Found: C, 68.13; H, 5.30.

2-Dihydro 4-oxo-[1,3-a]-naphthodioxane (6a). $1 \mathrm{~d},(\mathrm{Rf}=0.40),(62 \%)$, cream solid, m.p. 208$209{ }^{\circ} \mathrm{C}$. IR (Nujol) 3329.1m, 2855.0s, 1753.2m, 1729.7m, 1712.0m, $1694.1 \mathrm{~m}, 1661.0 \mathrm{~m}$, $1631.3 \mathrm{~m}, 1578.4 \mathrm{~m}, 1536.5 \mathrm{~m}, 1513.9 \mathrm{~m}, 1463.6 \mathrm{~s}, 1377.2 \mathrm{~s}, 1352.7 \mathrm{~m}, 1282.2 \mathrm{~m}, 1254.1 \mathrm{w}$, $1220.6 \mathrm{~m}, 1151.8 \mathrm{~m}, 1102.8 \mathrm{~m}, 1048.3 \mathrm{~m}, 1029.7 \mathrm{~m}, 980.3 \mathrm{~m}, 922.1 \mathrm{w}, 861.7 \mathrm{w}, 820.9 \mathrm{~m}, 792.2 \mathrm{~m}$, 762.2s, 722.4m, 698.1w, 647.9w cm ${ }^{-1} ;{ }^{1} \mathrm{H}$ NMR (300 MHz, $\left.\mathrm{CDCl}_{3}\right) \delta 8.17$ (d, J=7.59 Hz, $1 \mathrm{H}$, H5), 7.87 (d, J=8.69 Hz, 1H, H6), 7.84 (d, J=7.95 Hz, 1H, H10), 7.66 (d, J=1.27 Hz, 1H, H7), 7.58 (m, 2H, H8\&H9), 5.84 (s, 2H, $\left.\mathrm{CH}_{2}\right) ;{ }^{13} \mathrm{C}$ NMR (50 MHz, $\left.\mathrm{CDCl}_{3}\right) \delta 161.6(\mathrm{C}=\mathrm{O}), 156.9(\mathrm{C}-$ Ar), 137.2 (C-Ar), 130.1 (C5), 127.9 (C6), 126.9 (C10), 123.7 (C7), 123.0 (C8), 129.9 (C-Ar), 122.7 (C9), 109.0 (C-Ar), 91.2 (C2); HRMS calc. for $\mathrm{C}_{12} \mathrm{H}_{8} \mathrm{O}_{3}: \mathrm{m} / \mathrm{z}$ 200.0473, found 200.0488; $\mathrm{MS} m / \mathrm{z} 201(\mathrm{M}+1)^{+}$.

2-Ethyl 4-oxo-[1,3-a]-naphthodioxane (6b). $2 \mathrm{~d}$, ( $\mathrm{Rf}=0.42),(38 \%)$, colourless crystals (diethyl ether), m.p. $81-82{ }^{\circ} \mathrm{C}$. IR (Nujol) 2846.7s, 2724.6w, 1759.2m, 1721.2m, 1631.2m, 1581.3m, $1513.9 \mathrm{~m}, 1463.3 \mathrm{~s}, 1376.8 \mathrm{~s}, 1311.3 \mathrm{~m}, 1281.3 \mathrm{~m}, 1247.7 \mathrm{~m}, 1213.1 \mathrm{~m}, 1141.7 \mathrm{w}, 1020.6 \mathrm{~m}, 961.5 \mathrm{~m}$, 934.9m, 889.8m, 821.1m, 791.8m, 763.2m, 722.3m cm $\mathrm{m}^{-1}$; ${ }^{1} \mathrm{H}$ NMR (200 MHz, $\left.\mathrm{CDCl}_{3}\right) \delta 8.25$ (d, $\mathrm{J}=0.62 \mathrm{~Hz}, 1 \mathrm{H}, \mathrm{H} 5), 7.87$ (dd, J=8.69, $6.18 \mathrm{~Hz}, 2 \mathrm{H}, \mathrm{H} 6 \& H 10), 7.68$ (d, J=1.48 Hz, 1H, H7), 7.59 (m, 2H, H8\&H9), 5.75 (t, J=4.93, $4.97 \mathrm{~Hz}, 1 \mathrm{H}, \mathrm{H} 2), 2.23\left(\mathrm{~m}, 2 \mathrm{H}, \mathrm{CH}_{2}\right), 1.25$ (t, J=7.47, 7.56 Hz, $\left.3 \mathrm{H}, \mathrm{CH}_{3}\right) ;{ }^{13} \mathrm{C} \mathrm{NMR}\left(50 \mathrm{MHz}, \mathrm{CDCl}_{3}\right) \delta 162.7(\mathrm{C}=\mathrm{O}), 156.8$ (C-Ar), 137.3 (C-Ar), 129.9 (C5), 127.9 (C10), 126.8 (C6), 123.7 (C7), 123.1 (C9), 122.8 (C-Ar), 122.7 (C8), 108.6 (C-Ar), 102.5 (C2), $26.9\left(\mathrm{CH}_{2}\right), 7.4\left(\mathrm{CH}_{3}\right) ; \mathrm{MS} \mathrm{m} / \mathrm{z} 228$ (11), 171 (15), 170 (100), 115 (19), 114 (91), 113 (27), 88 (18), 63 (17); Anal. Calc. for $\mathrm{C}_{14} \mathrm{H}_{12} \mathrm{O}_{3}$ : C, 73.67. H, 5.30. Found: C, 73.87; H, 5.38.

2-Isopropyl 4-oxo-[1,3-a]-naphthodioxane (6c). $2 \mathrm{~d}$, ( $\mathrm{Rf}=0.40),(32 \%)$, colourless crystals (diethyl ether), m.p. $69-70{ }^{\circ} \mathrm{C}$. IR (Nujol) 2924.4s, $1757.9 \mathrm{~m}, 1720.6 \mathrm{~m}, 1630.0 \mathrm{~m}, 1581.0 \mathrm{~m}$, $1513.7 \mathrm{~m}, 1462.8 \mathrm{~s}, 1377.0 \mathrm{~s}, 1294.0 \mathrm{~m}, 1277.0 \mathrm{~m}, 1248.5 \mathrm{~m}, 1217.1 \mathrm{~m}, 1133.8 \mathrm{~m}, 1111.0 \mathrm{~m}$, $1051.7 \mathrm{~m}, 1020.1 \mathrm{~m}, 984.2 \mathrm{~m}, 957.7 \mathrm{~m}, 867.0 \mathrm{w}, 820.4 \mathrm{~m}, 790.1 \mathrm{~m}, 724.3 \mathrm{~m}, 649.3 \mathrm{w} \mathrm{cm}{ }^{-1}$; ${ }^{1} \mathrm{H}$ NMR $\left(200 \mathrm{MHz}, \mathrm{CDCl}_{3}\right) \delta 8.23$ (d, J=0.85 Hz, 1H, H5), 7.88 (dd, J=8.66, 7.67 Hz, 2H, H6\&H10), 7.70 (d, J=1.46 Hz, 1H, H7), 7.58 (m, 2H, H8\&H9), 5.56 (d, J=4.50 Hz, 1H, H2), 2.41 (m, 1H, CH), $1.26(\mathrm{~d}, \mathrm{~J}=5.27 \mathrm{~Hz}, 6 \mathrm{H}, 2 \mathrm{xCH})_{3} ;{ }^{13} \mathrm{C} \mathrm{NMR}\left(50 \mathrm{MHz}, \mathrm{CDCl}_{3}\right) \delta 162.8(\mathrm{C}=\mathrm{O}), 156.9(\mathrm{C}-\mathrm{Ar}), 137.3$ (C-Ar), 129.9 (C5), 128.0 (C10), 126.8 (C6), 123.8 (C7), 123.2 (C9), 122.7 (C-Ar), 122.6 (C8), 108.6 (C-Ar), 104.9 (C2), 31.9 (CH- $\left.{ }^{\mathrm{i}} \mathrm{Pr}\right), 16.3\left(\mathrm{CH}_{3}\right), 16.2\left(\mathrm{CH}_{3}\right)$; MS m/z 242 (9), 171 (15), 170 (100), 115 (12), 114 (43), 113 (12), 72 (9); Anal. Calc. for $\mathrm{C}_{15} \mathrm{H}_{14} \mathrm{O}_{3}$ : C, 74.36. H, 5.82. Found: C, 74.14; H, 5.71 .

2-Butyl 4-oxo-[1,3-a]-naphthodioxane (6d). $2 \mathrm{~d}$, ( $\mathrm{Rf}=0.35),(38 \%)$, colourless crystals (diethyl ether), m.p. $60-62{ }^{\circ} \mathrm{C}$. IR (Nujol) 2851.0s, $1757.7 \mathrm{~s}, 1633.1 \mathrm{~m}, 1582.1 \mathrm{~m}, 1514.0 \mathrm{~m}, 1463.2 \mathrm{~s}$, $1376.9 \mathrm{~s}, 1278.6 \mathrm{~m}, 1208.9 \mathrm{~m}, 1138.6 \mathrm{w}, 1108.3 \mathrm{w}, 1021.8 \mathrm{w}, 970.2 \mathrm{w}, 823.8 \mathrm{~m}, 793.3 \mathrm{w}, 762.5 \mathrm{~m}$, $722.3 \mathrm{~m} \mathrm{~cm}^{-1}$; ${ }^{1} \mathrm{H}$ NMR $\left(200 \mathrm{MHz}, \mathrm{CDCl}_{3}\right) \delta 8.24(\mathrm{~d}, \mathrm{~J}=0.66 \mathrm{~Hz}, 1 \mathrm{H}, \mathrm{H} 5), 7.87$ (dd, J=8.67, 5.94 Hz, 2H, H6\&H10), 7.84 (d, J=1.51 Hz, 1H, H7), 7.63 (m, 2H, H8\&H9), 5.78 (t, J=5.1, 5.14 Hz, $1 \mathrm{H}, \mathrm{H} 2), 2.20\left(\mathrm{~m}, 2 \mathrm{H}, \mathrm{CH}_{2}\right), 1.66\left(\mathrm{~m}, 2 \mathrm{H}, \mathrm{CH}_{2}\right), 1.48\left(\mathrm{~m}, 2 \mathrm{H}, \mathrm{CH}_{2}\right), 1.00$ (t, J=7.17, $7.21 \mathrm{~Hz}, 3 \mathrm{H}$, 
$\left.\mathrm{CH}_{3}\right) ;{ }^{13} \mathrm{C}$ NMR $\left(50 \mathrm{MHz}, \mathrm{CDCl}_{3}\right) \delta 162.6(\mathrm{C}=\mathrm{O}), 156.7$ (C-Ar), 137.2 (C-Ar), 129.9 (C5), 127.9 (C10), 126.7 (C6), 123.7 (C7), 123.1 (C-Ar), 122.6 (C9), 122.3 (C8), 108.6 (C-Ar), 101.8 (C2), $33.2\left(\mathrm{CH}_{2}\right), 25.1\left(\mathrm{CH}_{2}\right), 22.3\left(\mathrm{CH}_{2}\right), 13.8\left(\mathrm{CH}_{3}\right) ; \mathrm{MS} \mathrm{m} / \mathrm{z} 256$ (16), 171 (16), 170 (100), 115 (11), 114 (42), 113 (10); Anal. Calc. for $\mathrm{C}_{16} \mathrm{H}_{16} \mathrm{O}_{3}$ : C, 74.98. H, 6.29. Found: C, 75.05; H, 6.37.

2-Isopropylidene glyceral 4-oxo-[1,3-a]-naphthodioxane (8). $1 \mathrm{~d},(\mathrm{Rf}=0.22),(65 \%)$, colourless crystals (diethyl ether), m.p. $105-106^{\circ} \mathrm{C},[\alpha]_{\mathrm{D}}{ }^{0} 4.9\left(\mathrm{C} 3, \mathrm{CHCl}_{3}\right)$. IR (Nujol) $3329.6 \mathrm{~m}$, $2922.4 \mathrm{~s}, 2853.8 \mathrm{~s}, 1729.4 \mathrm{~m}, 1711.7 \mathrm{~m}, 1694.1 \mathrm{~m}, 1680.9 \mathrm{~m}, 1536.4 \mathrm{~m}, 1462.7 \mathrm{~s}, 1377.0 \mathrm{~s}, 1291.0 \mathrm{w}$, $1254.0 \mathrm{~m}, 1164.6 \mathrm{w}, 1054.3 \mathrm{w}, 979.6 \mathrm{~m}, 763.7 \mathrm{w}, 722.2 \mathrm{~m}, 702.0 \mathrm{w} \mathrm{cm}^{-1} ;{ }^{1} \mathrm{H}$ NMR $(200 \mathrm{MHz}$, $\left.\mathrm{CDCl}_{3}\right) \delta 8.22(\mathrm{~d}, \mathrm{~J}=0.90 \mathrm{~Hz}, 1 \mathrm{H}, \mathrm{H} 5), 7.89$ (m, 2H, H6\&H10), 7.72 (d, J=5.43 Hz, 1H, H7), 7.63 (m, 2H, H8\&H9), 5.83 (d, J=4.09 Hz, 1H, H2), $4.62(\mathrm{~m}, 1 \mathrm{H}, \mathrm{CH}), 4.38\left(\mathrm{~m}, 2 \mathrm{H}, \mathrm{CH}_{2}\right), 1.55(\mathrm{~d}$, $\left.\mathrm{J}=28.04 \mathrm{~Hz}, 6 \mathrm{H}, 2 \mathrm{xCH}_{3}\right) ;{ }^{13} \mathrm{C}$ NMR $\left(50 \mathrm{MHz}, \mathrm{CDCl}_{3}\right) \delta 161.4(\mathrm{C}=\mathrm{O}), 137.4(\mathrm{C}-\mathrm{Ar}), 130.2(\mathrm{C} 5)$, 128.1 (C10), 127.1 (C6), 123.7 (C7), 123.3 (C9), 123.1 (C-Ar), 122.7 (C8), 111.0 (C-Ar), 108.8 (C-Ar), $99.8(\mathrm{C} 2), 95.4(\mathrm{C}-\mathrm{i} P r), 74.9(\mathrm{CH}), 64.6\left(\mathrm{CH}_{2}\right), 26.5\left(\mathrm{CH}_{3}\right), 25.2\left(\mathrm{CH}_{3}\right)$; HRMS calc. for $\mathrm{C}_{17} \mathrm{H}_{16} \mathrm{O}_{5}: \mathrm{m} / \mathrm{z} 300.0998$, found 300.1008; MS m/z $301.1(\mathrm{M}+\mathrm{H})^{+}$.

\section{Acknowledgements}

E. P. thanks the Australian Agency for International Development for an overseas research scholarship.

\section{References}

1. Perlmutter, P.; Puniani, E. Tetrahedron Lett. 1996, 37, 1715.

2. Jackson, D. Y. Synth. Commun. 1988, 18, 337.

3. Kocienski, P. J. Protecting Groups Thieme: Stuttgart, 1994.

4. Khan, A. A.; Emslie, N. D.; Drewes, S. E.; Field, J. S.; Ramesar, N. Chem. Ber. 1993, 126, 1477.

5. Evans, J. C.; Klix, R. C.; Bach, R. D. J. Org. Chem. 1988, 53, 5519.

6. Jadhav, G. V.; Thakkar, R. M. J. Univ. Bombay Sect. A 1949, 18, 29. 